\title{
Chemical Exchange Saturation Transfer in Chemical Reactions: A Mechanistic Tool for NMR Detection and Characterization of Transient Intermediates
}

\author{
N. Lokesh, Andreas Seegerer, Johnny Hioe, and Ruth M. Gschwind*(i) \\ Institute of Organic Chemistry, University of Regensburg, D-93053 Regensburg, Germany
}

Supporting Information

ABSTRACT: The low sensitivity of NMR and transient key intermediates below detection limit are the central problems studying reaction mechanisms by NMR. Sensitivity can be enhanced by hyperpolarization techniques such as dynamic nuclear polarization or the incorporation/interaction of special hyperpolarized molecules. However, all of these techniques require special equipment, are restricted to selective reactions, or undesirably influence the reaction pathways. Here, we apply the chemical exchange saturation transfer (CEST) technique for the first time to NMR detect and characterize previously unobserved transient reaction intermediates in organocatalysis. The higher sensitivity of CEST and chemical equilibria present

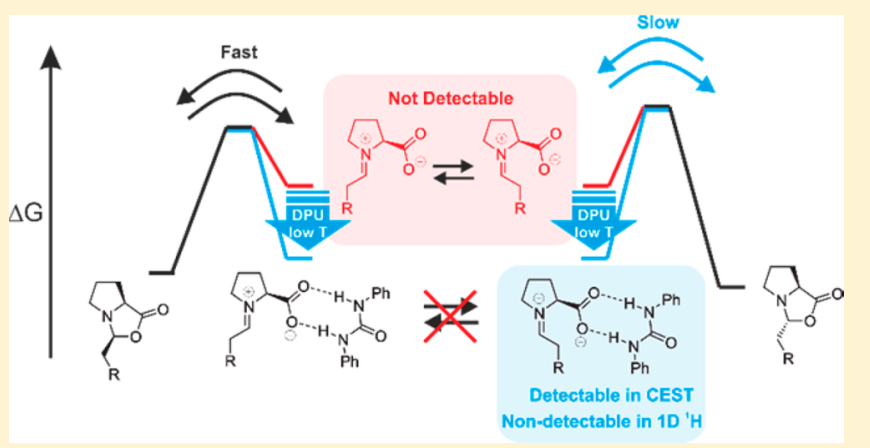
in the reaction pathway are exploited to access population and kinetics information on low populated intermediates. The potential of the method is demonstrated on the proline-catalyzed enamine formation for unprecedented in situ detection of a DPU stabilized zwitterionic iminium species, the elusive key intermediate between enamine and oxazolidinones. The quantitative analysis of CEST data at $250 \mathrm{~K}$ revealed the population ratio of $[Z$-iminium $] /[$ exo-oxazolidinone] 0.02 , relative free energy +8.1 $\mathrm{kJ} / \mathrm{mol}$ (calculated $+7.3 \mathrm{~kJ} / \mathrm{mol}$ ), and free energy barrier of $+45.9 \mathrm{~kJ} / \mathrm{mol}\left(\Delta G_{\text {calc. }}^{\ddagger}(268 \mathrm{~K})=+42.2 \mathrm{~kJ} / \mathrm{mol}\right)$ for $Z$-iminium $\rightarrow$ exo-oxazolidinone. The findings underpin the iminium ion participation in enamine formation pathway corroborating our earlier theoretical prediction and help in better understanding. The reliability of CEST is validated using 1D EXSY-build-up techniques at low temperature $(213 \mathrm{~K})$. The CEST method thus serves as a new tool for mechanistic investigations in organocatalysis to access key information, such as chemical shifts, populations, and reaction kinetics of intermediates below the standard NMR detection limit.

\section{INTRODUCTION}

NMR spectroscopy is a key method to reveal and interpret reaction mechanisms in chemical and catalytic reactions due to its unique potential to provide experimental information about structures, aggregation, and interactions in solution. ${ }^{1-7}$ However, the main drawback of NMR spectroscopy is its inherent insensitivity compared to other methods like UV-vis or mass spectrometry, which often limits its applicability. For the mechanistic investigation of chemical reactions by NMR, this drawback is especially serious, since in most reactions the intermediates, which are key to the reaction mechanism, are low populated, short-lived, and most often below the detection limit. Failure in observing such key intermediates (missing evidence) often leads to ambiguity in understanding the reaction pathway. The theoretical calculations can assist in predicting possible mechanistic pathways, however, the experimental detection of more active intermediates and its kinetic data during the reaction is essential to pinpoint the most plausible pathway.

One notable example is the proline or proline derivativemediated enamine catalysis, which evolved as a versatile tool in a broad scope of enantioselective transformations of carbonyl compounds. $^{8-10}$ However, for almost a decade, an in situ detection of the central enamine intermediate in prolinecatalyzed aldol reaction was lacking, leading to controversial discussions about its formation, ${ }^{11-18}$ stabilization, ${ }^{4,19}$ and the subsequent transformation pathways. In the earlier stage, Seebach and Eschenmoser suggested that the observed oxazolidinone exists in equilibrium with the enamines (Figure 1). ${ }^{14}$ In 2010 our group was able to NMR detect and characterize the in situ generated enamine intermediate through solvent/additive-induced stabilization. ${ }^{4,16,19,20}$ The observed chemical exchange between oxazolidinone and enamine and the missing evidence for iminium ion were strongly indicative for an enamine formation via oxazolidinone (referred as oxazolidinone pathway, Figure 1). ${ }^{14}$ Further, this was also supported by recent kinetic isotope effect measurements for a L-proline-catalyzed $\alpha$-amination of 3-phenylpropionalaldehyde. $^{21}$ In contrast, List and Houk proposed an enamine formation via zwitterionic iminium ion intermediates (referred as iminium pathway, Figure 1), 9,12,17,18 supported by DFT-

Received: November 22, 2017

Published: January 16, 2018 


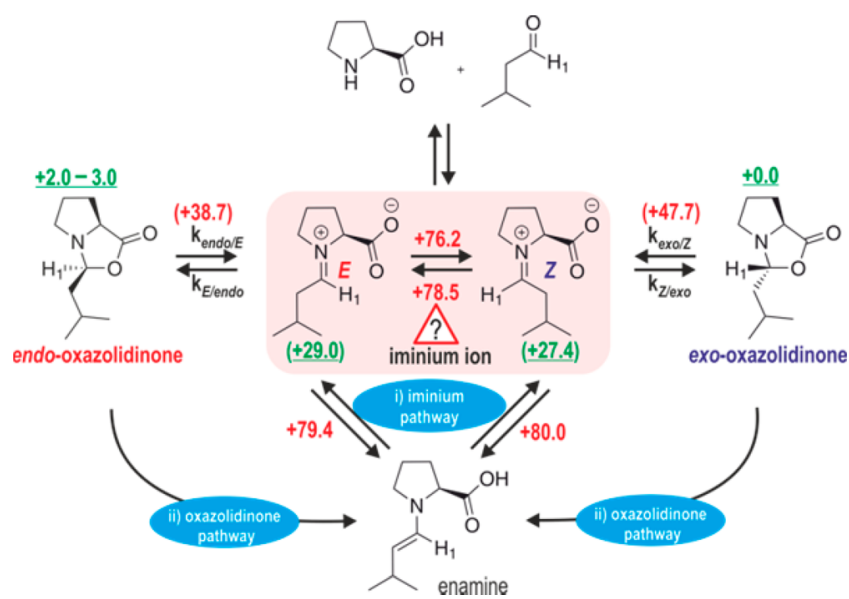

Figure 1. Formation of enamine, the central iminium ion intermediates are elusive. (i) Iminium pathway: formation of enamine by deprotonation of an iminium ion; (ii) oxazolidinone pathway: direct formation of enamine by deprotonation of a oxazolidinone intermediate. The energy values shown are obtained from theoretical calculations (with parentheses) and experiments (without parentheses). ${ }^{15}$ Green (underlined): Relative free energy to exooxazolidinone in DMSO at $300 \mathrm{~K}$. Red: Free energy barrier predicted by theory and measured from EXSY-experiment in DMSO at $300 \mathrm{~K}^{15}$ All calculations were performed without any external base.

calculations. ${ }^{11}$ The recent kinetic studies in our working group $^{15}$ revealed that enamine formation rate and barrier conform to the calculated pathway via iminium intermediate. Furthermore, the barriers of the ring opening step from oxazolidinone toward the iminium intermediate are generally much lower than the proton abstraction to form enamine and exo $\leftrightarrow$ endo interconversion via double-bond rotation (Figure 1)..$^{15}$

Despite the quantitative agreement to the kinetic data, the existence of such unstable zwitterionic intermediates remains intriguing. Recent higher level theoretical calculation showed that the $E / Z$-iminium intermediates are indeed located about 27-30 $\mathrm{kJ} / \mathrm{mol}$ higher than the corresponding endo/exooxazolidinone even in high polar medium. ${ }^{15}$ According to Boltzmann distribution, this corresponds to a population of $0.03 \%$ relative to oxazolidinones $(\mu \mathrm{M}$ amount in reaction condition), which is unlikely to be detected by conventional NMR. To prove the possible participation of iminium ions in this L-proline-catalyzed reaction, it necessitates more sensitive NMR techniques and/or stabilization methods to increase its amount.

Hyperpolarization techniques such as dissolution $\mathrm{DNP},{ }^{22-24}$ PHIP, ${ }^{25-27}$ SABRE, ${ }^{25-27}$ and CIDNP/photo CIDNP ${ }^{28-31}$ are used to enhance the sensitivity of the NMR signals. However, despite successful detection of low concentrated nuclei, ${ }^{32}$ the hyperpolarization techniques lacks generality due to additional requirements, such as radicals and microwave in DNP, para hydrogen in PHIP and SABRE, and radical pair presence in CIDNP or photo-CIDNP, which are suitable only to specific reactions or reaction conditions and often alters the chemical pathways. Furthermore, use of cryo-probes can enhance sensitivity, however these are incompatible at low temperatures, which are most often necessary for intermediates stabilization. ${ }^{1,3}$

In this regard, the chemical exchange saturation transfer (CEST) technique has attracted immense interest. The method provides more than 10 -fold sensitivity enhancement, ${ }^{33,34}$ which is moderate compared to hyperpolarization techniques, but more importantly it does not influence the reaction system as it does not require external agents, such as radicals, para hydrogen etc. For CEST the only requirements are the existence of a chemical exchange in the slow exchange regime on the NMR time scale $(k \leq|\Delta \nu|)$ and one of the chemical exchange species being visible in conventional NMR experiments. ${ }^{35}$ CEST can then detect a low populated conformer or species below the conventional NMR detection limit. This is achieved by saturation transfer of selectively saturated nuclei from low populated site to major populated site via chemical/conformational exchange. During the saturation process, more numbers of saturated nuclei from a low populated site accumulate at a major populated site resulting in a decrease in intensity of major populated nuclei peak, which is more amplified than direct detection of low populated nuclei. ${ }^{35}$ The CEST method does not require any pre-knowledge of chemical shift information, as the method involves a stepwise systematic scanning of the chemical shift regions by selective saturation. The decrease in intensity as a function of saturation offset (chemical shift) provides information about relative population, structure, and rate of exchange of saturated molecule via Bloch-McConnell equations. ${ }^{36-38}$ However, CEST also shows a decrease in intensity due to NOE interactions as well, which often complicates the study. A few methods were reported which eliminates NOE interactions. ${ }^{39,40}$ In addition, various CEST modules were developed, such as, proton detected, heteronuclear detected, and multiple quantum-based methods to avoid overlapping, cover a broad range of exchange rates, and accelerate the experiment. ${ }^{41-45}$

Initial application of CEST was shown in MRI as an alternative contrast imaging technique. ${ }^{46-48}$ In this, the exchangeable protons in exogenous/endogenous compounds are selectively saturated and are detected indirectly through an intensity decrease in the water signal due to saturation transfer, which gives enhanced sensitivity even for low concentrated molecules. ${ }^{33,34}$ Recently, the signal amplification obtained in CEST is exploited for very compelling application in detecting invisible conformations of biomolecules, such as proteins and nucleic acids, which were never detected in conventional NMR experiments before. ${ }^{43,45,49}$ Despite the broad application of the CEST technique for the study of protein conformations and its high potential to reveal invisible states, to the best of our knowledge, the method has never been employed in reaction intermediate studies of chemical or catalytic reactions so far.

Here, we present for the first time CEST application for detection and characterization of low populated transient reaction intermediates in chemical and catalytic reactions, which are not visible in conventional NMR experiments. The advantage of signal amplification obtained in CEST and present chemical equilibrium in reaction pathway are explored, to prove for the first time in situ existence of iminium ion intermediates in enamine catalysis, which are key intermediates in the enamine formation pathway. Furthermore, the population of the invisible intermediate, its thermal stability, and free energy barrier for ring opening and closing are obtained through quantitative analysis of CEST profiles. At low temperature, the reliability of the CEST data about energies and exchange rates is validated by $1 \mathrm{D}$ EXSY and computational studies. The detection of such thermodynamically unstable intermediates underpins the formation pathway via iminium ion. 


\section{RESULTS AND DISCUSSION}

In the following it is described how even a complicated multisite equilibrium in a reaction can be prepared/adapted to the successful application of CEST. For this, mainly three points have to be addressed (i) absolute population of the elusive intermediate, (ii) slow exchange, and (iii) reduction of multisite equilibria. Without additives, slow exchange and two site equilibria can be reached at low temperatures, but the population is still too low (see CEST without Stabilizing Agent). Upon addition of an additive, its effect on the chemical shifts and the intermediate structures is analyzed (see DPU as Stabilizing Agent at $300 \mathrm{~K}$ ). Applying an additive and low temperature, all three requirements for CEST are fulfilled, and theoretical calculations show that the additive does not significantly alter the kinetics of the investigated part of the reaction (see DPU as Stabilizing Agent at Low Temperature) allowing its application to detect the elusive iminium intermediate (see Detection of Z-Iminium via CEST). The reduction of multisite equilibrium to two site equilibrium allows for the quantitative determination of thermodynamic and kinetic parameters by CEST (see Population and Kinetics via CEST Profile). Finally, at very low temperatures, the CEST results were validated by classical $1 \mathrm{D}$ EXSY rate measurements, further the benefits of CEST are highlighted over EXSY (see Validation of CEST Method).

Model System. To successfully illustrate the potential of CEST for detection and characterization of elusive iminium intermediates, an appropriate model system has to be chosen, which allows simultaneous detection of oxazolidinone diastereomers and enamine intermediates in the reaction by conventional NMR and shows slow chemical exchanges between the intermediates. Based on our earlier experience, the L-proline-catalyzed homo aldol condensation of 3methylbutanal fulfills these requirements (Figure 1). ${ }^{4,15,50}$ In addition, the formed intermediates, that is, endo/exo-oxazolidinones, enamines, and the substrates, give the best signal separation in NMR for proton $\mathrm{H}_{1}$ (Figure 2). The proton $\mathrm{H}_{1}$ of

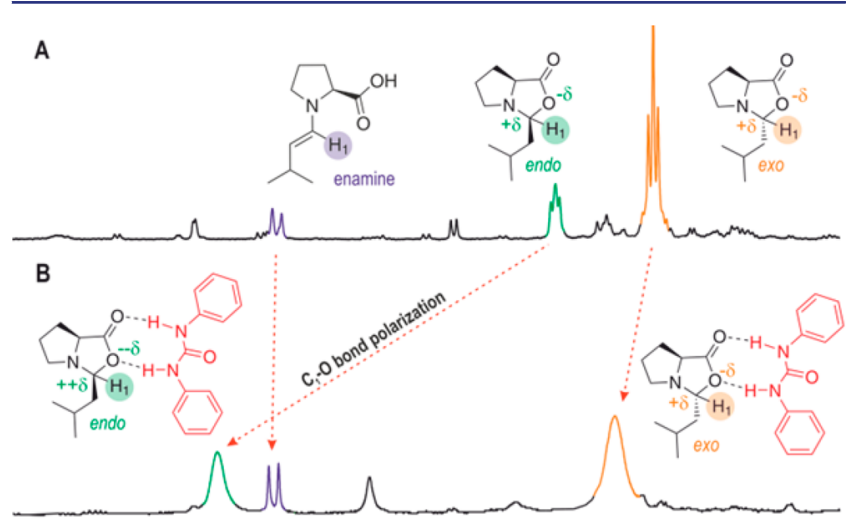

$\begin{array}{lllllllllllllllllll}6.7 & 6.6 & 6.5 & 6.4 & 6.3 & 6.2 & 6.1 & 6.0 & 5.9 & 5.8 & 5.7 & 5.6 & 5.5 & 5.4 & 5.3 & 5.2 & 5.1 & 5.0 & \mathrm{ppm}\end{array}$

Figure 2. $1 \mathrm{D}{ }^{1} \mathrm{H}$ NMR spectra of the reaction mixture of L-proline and 3-methylbutanal in DMF- $d_{7}$ at $300 \mathrm{~K}$ without (A) and with DPU (B).

the elusive iminium ion is expected to be found well-separated at around $9 \mathrm{ppm}$ based on our theoretical calculations and earlier reports. $^{51,52}$ Thus, CEST selective saturation around 9 ppm should cause an intensity decrease for the $\mathrm{H}_{1}$ signal of the oxazolidinones and/or enamines if they are in slow exchange with the elusive iminium intermediates (Figure 1).
CEST without Stabilizing Agent. The recorded 1D ${ }^{1} \mathrm{H}$ NMR spectrum for the reaction shows well-separated signals of the $\mathrm{H}_{1}$ proton for all three detectable intermediates, enamine, exo- and endo-oxazolidinone (see highlighted signals in Figure 2A). As expected, the stable oxazolidinone intermediates are observed in $1 \mathrm{D}{ }^{1} \mathrm{H}$ NMR (Figure 2), but due to the higher energy, the iminium ion intermediates were not observed. The approximate population of intermediates formed in the investigated reaction are $4 \mathrm{mM}, 1.8 \mathrm{mM}$, and $0.7 \mathrm{mM}$, respectively, for exo-, endo-oxazolidinone and enamine. According to our previous calculation in DMSO, the oxazolidinone is $27-30 \mathrm{~kJ} / \mathrm{mol}$ more stable than the iminium intermediates, ${ }^{15}$ which refers to a population of iminium ions $<0.03 \%$ or equivalently $1.2 \mu \mathrm{M}$ in the reaction. This is clearly below the threshold of conventional NMR spectroscopy. However, even CEST measurements with much higher sensitivity (more than 10-fold) at $300 \mathrm{~K}$ do not show any decrease in intensities for the $\mathrm{H}_{1}$-protons of oxazolidinones/ enamines on saturating around $9 \mathrm{ppm}$.

The thermal instability of the iminium intermediates seems to be one of the problems to be solved. Additionally, the calculated activation energy for the ring opening process is low (in DMSO at $300 \mathrm{~K}$, exo $\rightarrow Z=47.7 \mathrm{~kJ} / \mathrm{mol}$; endo $\rightarrow E=38.7$ $\mathrm{kJ} / \mathrm{mol}$; Figure 1), therefore we expect fast exchange. A common strategy to circumvent both issues is by reducing the temperature to stabilize the charge separated species (i.e., in this case the zwitterionic iminium ions, by increasing the dielectric constant of the medium) and to slow down exchange. Therefore, DMF is used in the investigation, since it is highly polar, similar to DMSO, and also allows measurement at low temperature up to $213 \mathrm{~K}$.

However, the low-temperature 1D ${ }^{1} \mathrm{H}$ NMR, 1D EXSY, and CEST experiments down to $215 \mathrm{~K}$ did not give any evidence for iminium ion intermediates even though the system is expected to be in the slow exchange regime (see Figure S1 in Supporting Information for spectra without DPU). This implies that the stabilization of charge separated species by increasing dielectric constant is not sufficient to alter the thermal population for the detection even in CEST.

DPU as Stabilizing Agent at $\mathbf{3 0 0} \mathrm{K}$. Therefore, next the iminium population was increased by an additive. It was shown in literature that addition of thiourea derivatives could possibly stabilize proline iminium ion species. ${ }^{53}$ As also shown in earlier $^{19,50}$ and current studies in DMSO/DMF, a significant downfield shift of $\mathrm{H}_{1}$ endo-oxazolidinone proton $(\Delta \delta=0.8$ $\mathrm{ppm})$ upon the addition of 1 equiv of DPU $\left(N, N^{\prime}\right.$-diphenyl urea) to the investigated reaction mixture was observed (Figure 2B). In contrast, only a slight shift for exo-oxazolidinone $(\Delta \delta=$ $0.1 \mathrm{ppm})$, and nearly no shift for the enamine peak is observed. ${ }^{19,50}$ For these observations, there are several possible roots, which could induce the shift at $300 \mathrm{~K}$ when DPU is added. The first obvious one is the deshielding of $\mathrm{H}_{1}$ by the aromatic moiety of the DPU, which might be different for endoand exo-oxazolidinone. However, from our detailed structural analysis, no particular effect is expected since the aromatic ring is remotely located relative to $\mathrm{H}_{1}$ of both oxazolidinones (see Supporting Information).

The second possible reason is due to the formation of significant iminium species along with the oxazolidinones, which could be in fast exchange with each other leading to downfield averaged peak for $\mathrm{H}_{1}{ }^{50}$ In this case, the peak location is determined by the population percentage of the two exchanging molecules, that is, endo $\leftrightarrow E$ and exo $\leftrightarrow Z$ 
(considering negligible exchange between exo and endo). However, EXSY measurement at $300 \mathrm{~K}$ revealed similar interconversion rates for endo $\rightarrow$ exo $(+72.5 \mathrm{~kJ} / \mathrm{mol})$ and exo $\rightarrow$ endo $(+72.9 \mathrm{~kJ} / \mathrm{mol})$ (Figure 3$)$. This indicates a

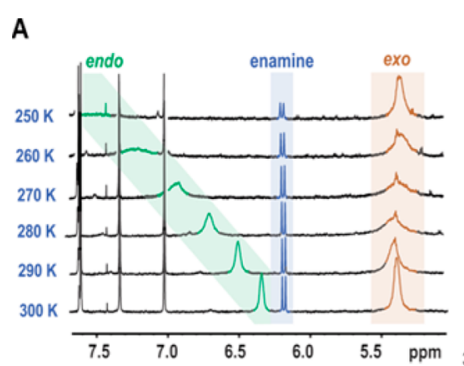

\section{B}

C

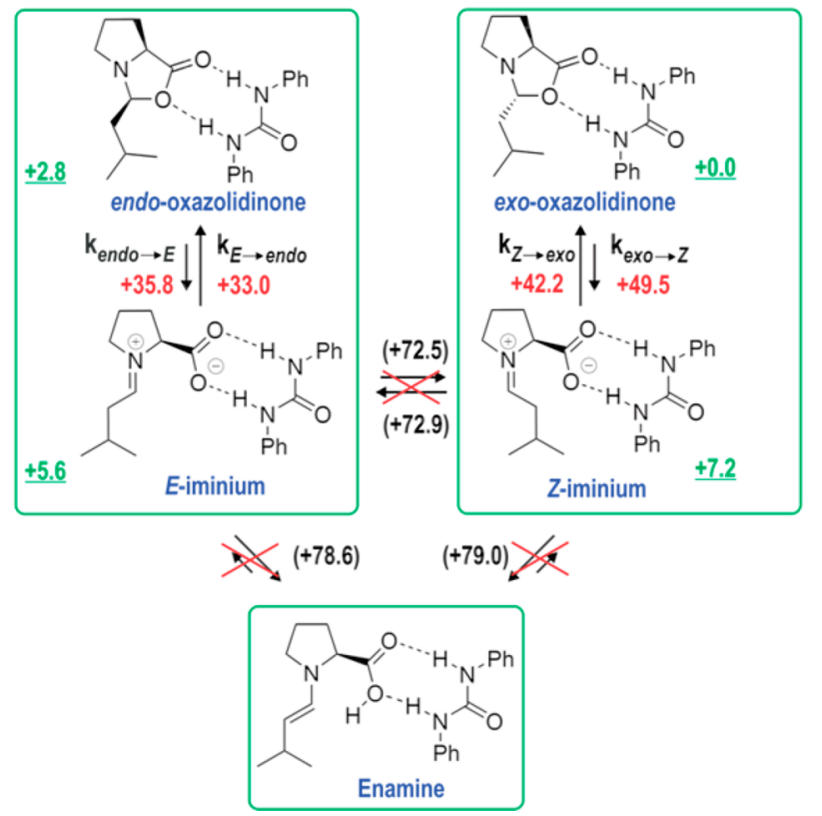

Figure 3. Temperature-dependent stacked ${ }^{1} \mathrm{H} 1 \mathrm{D}$ spectra (A) with decrease in temperature $\mathrm{H}_{1}$ of endo-oxazolidinone peak shows a line broadening and downfield shift, and $\mathrm{H}_{1}$ of exo-oxazolidinone shows a narrower-broader-narrower profile indicating shift of exo $\leftrightarrow Z$ exchange to slow exchange regime. Temperature-dependent plot of $\mathrm{H}_{1}$ peak shift of exo- and endo-oxazolidinone and enamine in the presence of DPU (the first points at $300 \mathrm{~K}$ are without DPU) (B). At low temperature, no chemical exchange occurs between exo- and endooxazolidinone via iminium $(T<250 \mathrm{~K})$ as well as oxazolidinones and the enamine $(T<270 \mathrm{~K})$. Numbers $(\mathrm{kJ} / \mathrm{mol})$ indicated in black with parentheses: experimental free energy barrier from EXSY at $300 \mathrm{~K}$; red: calculated free energy barrier at $268 \mathrm{~K}$; green (underlined): calculated relative Gibbs free energy at $268 \mathrm{~K}$ (C).

thermodynamic equivalency between $E$ - and $Z$-iminium. Furthermore, the population analysis of endo and exo (peak integration) showed a relatively higher free energy for endooxazolidinone $(2.0 \mathrm{~kJ} / \mathrm{mol})$, which puts $E$-iminium energetically nearer to the endo-oxazolidinone. Hence, one might tend to expect the more pronounced shift observed for $\mathrm{H}_{1}$ of endooxazolidinone. Nevertheless, as it will be indicated later by $\mathrm{CEST}$, at $300 \mathrm{~K}$ the population of the $E / Z$-iminium is expected to be very low $\left([Z\right.$-iminium $] /[$ exo $]<2 \%$ or $\Delta G_{\text {-exo }}(300 \mathrm{~K})>$ $9.5 \mathrm{~kJ} / \mathrm{mol} ; E$-iminium $/[$ endo $]<5 \%$ or $\Delta G_{E \text {-endo }}(300 \mathrm{~K})>7.5$ $\mathrm{kJ} / \mathrm{mol}$ ). Given an expected $\mathrm{H}_{1}$ shift of the iminium species located at $\sim 9 \mathrm{ppm},{ }^{51,52}$ such low iminium population cannot contribute significantly to the observed average shift (maximum
$\Delta \delta=0.18 \mathrm{ppm}$ shift in case of $5 \% E$-iminium population and 95\% endo-oxazolidinone).

A third possible explanation is provided by the charge and structural analysis of oxazolidinones. Detailed structural analysis revealed that the $\mathrm{C}_{1}-\mathrm{O}$ bond is elongated by the complexation with DPU, however, more pronounced in endo-oxazolidinone than in exo-oxazolidinone (see Supporting Information; $\mathrm{H}_{1}$ is attached to $\mathrm{C}_{1}$ ). Consequently, the developed charge polarization along the $\mathrm{C}_{1}-\mathrm{O}$ bond in oxazolidinones causes the deshielding effect for $\mathrm{H}_{1}$ (stronger in endo, Figure 2B, Supporting Information). Therefore, contrary to the general expectation, the major effect observed at $300 \mathrm{~K}$ is not due to the population of iminium ion in the system, but rather due to the increased polarization of the $\mathrm{C}_{1}-\mathrm{O}$ bond.

At this point, the observed $\mathrm{H}_{1}$ chemical shift change only indicates a significant interaction between oxazolidinone and DPU. However, the detection and detailed characterization of iminium ions as well as the kinetics of its formation are not accessible due to low iminium ion population and too fast exchange at $300 \mathrm{~K}$.

DPU as Stabilizing Agent at Low Temperature. Therefore, the reaction mixture was cooled again to minimize the chemical exchange between the intermediates and to stabilize the iminium ion. On decreasing the temperature until $250 \mathrm{~K}$, a continued downfield shift and broadening for the $\mathrm{H}_{1}$ signal of endo-oxazolidinone is observed (Figure 3A,B). This significant shift is attributed to both $\mathrm{C}_{1}-\mathrm{O}$ polarization and interconversion between endo $\leftrightarrow E$-iminium ion. However, at low temperature, downfield shift is expected mainly due to increased population of zwitterionic E-iminium ion. Below 250 $\mathrm{K}$, the peak completely diminishes due to broadening (Figure $3 \mathrm{~A}$ ), and we were unable to reach the low exchange regime for the application of CEST (detailed estimation for the population of $E$-iminium ion and endo-oxazolidinone through classical peak analysis is shown in the Supporting Information). On the other hand, a slight upfield shift (Figure 3B) and line broadening on down to $280 \mathrm{~K}$ (Figure $3 \mathrm{~A}$, coalescence point) is observed for $\mathrm{H}_{1}$ signal of exo-oxazolidinone, indicating a possible exooxazolidinone $\leftrightarrow Z$-iminium exchange shift to the slow exchange regime. On further decreasing the temperature $(<280 \mathrm{~K})$, the broadened peak becomes narrower (Figure 3A). This narrower-broader-narrower profile confirms slow exchange regime for exo-oxazolidinone $\leftrightarrow Z$-iminium, which is a prerequisite for the application of CEST.

The quantum chemical calculations showed that the $E / Z$ iminium intermediates are massively stabilized by the presence of DPU due to the H-bond formation $\left(\Delta G_{268} Z\right.$-iminium vs exo $=+7.2 \mathrm{~kJ} / \mathrm{mol}$ and $\Delta G_{268}$ E-iminium vs endo $=+2.8 \mathrm{~kJ} / \mathrm{mol}$, Figure $3 \mathrm{C}$ ). Moreover, the calculated free energy difference between $E$ and $Z$ iminium at $268 \mathrm{~K}$ amounts to $1.6 \mathrm{~kJ} / \mathrm{mol}$ in favor of $E$-iminium. Surprisingly, the ring opening barriers were not affected significantly by the complexation with the additive $\left(\Delta G^{\ddagger}{ }_{268}=+35.8 \mathrm{~kJ} / \mathrm{mol}\right.$ for endo $\rightarrow E$ and $+49.5 \mathrm{~kJ} / \mathrm{mol}$ for exo $\rightarrow Z$, Figure 3C; see Figure 1 for barriers without DPU). At low temperature $(T \leq 250 \mathrm{~K})$, apart from the exchange between iminium and oxazolidinone, no exchange is possible between endo/exo $\leftrightarrow$ enamine and exo $\leftrightarrow$ endo or $Z \leftrightarrow E$ (1D ${ }^{1} \mathrm{H}$ EXSY showed no exchange peak, Figure 4B) due to the high barrier for deprotonation and double-bond rotation (Figure 3C: Measured $\Delta G^{\ddagger}{ }_{300}$ exo $\leftrightarrow$ endo and exo/endo $\leftrightarrow$ enamine; $\Delta G^{\ddagger}$ is quasiconstant over temperatures from 215 to $300 \mathrm{~K}$ ). Thus, we expect that the reduced thermal energy permits only the ring opening process to occur during $T_{1}$ time scale (Figure 

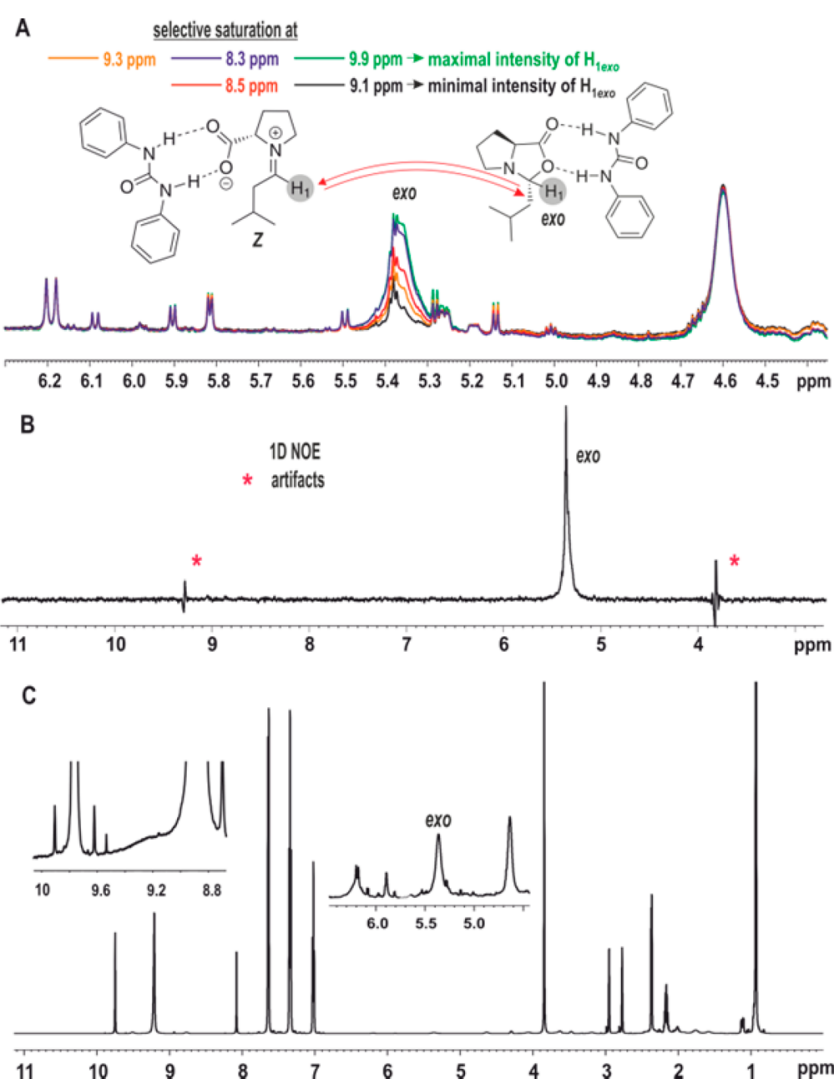

Figure 4. CEST (A), $1 \mathrm{D}{ }^{1} \mathrm{H}$ EXSY with mixing time $450 \mathrm{~ms}$ (B), and $1 \mathrm{D}{ }^{1} \mathrm{H}$ NMR (C) spectra at $250 \mathrm{~K}$. CEST shows decrease in intensity of exo-oxazolidinone due to saturation transfer from iminium, confirming the presence of iminium ions. EXSY shows no observation of iminium ion. Inset shows scaled exo-oxazolidinone intermediate peak from $1 \mathrm{D}{ }^{1} \mathrm{H}$ spectrum (C).

$3 \mathrm{C})$, the remaining exchange pathways are by far slow or nil and do not interfere with CEST application. Thus, the application of low temperature simplifies the complex multisite exchange system of the enamine catalysis into two site slow exchange equilibrium on CEST experimental time scale $\left(T_{1}\right.$ time scale), which allows a straightforward and reliable extraction of kinetic and thermodynamic information on iminium ion from CEST.

Detection of Z-Iminium via CEST. After successfully preparing the system for complying the prerequisites, slow exchange regime and two site equilibrium, the system is examined for iminium detection by CEST. The temperature was cooled stepwise, each step down by $10 \mathrm{~K}$. At each temperature, the 1D CEST experiments were recorded as described above, while selectively saturating around $9 \mathrm{ppm}$ and monitoring the intensity of the oxazolidinone peaks. Above 250 $\mathrm{K}$, no reduction was observed, but at $250 \mathrm{~K}$, the measured CEST experiment showed a decrease in intensity for the exooxazolidinone peak (Figure 4A). The decrease in intensity is pronounced as selective saturation approaches $9.12 \mathrm{ppm}$. At $9.12 \mathrm{ppm}$ a maximum drop in intensity was observed, which lessens as the selective saturation moves away from $9.12 \mathrm{ppm}$. This indicates the presence of a transient iminium ion intermediate at $9.12 \mathrm{ppm}$, which chemically exchanges with exo-oxazolidinone. As discussed above, the high energy barrier between exo- and endo-oxazolidinone (Figure 3C: $\Delta G^{\ddagger}{ }_{300}$ exo $\rightarrow$ endo $=+72.9 \mathrm{~kJ} / \mathrm{mol}$; endo $\rightarrow$ exo $=+72.5 \mathrm{~kJ} / \mathrm{mol}$ ) completely excludes interconversion between exo- and endo- oxazolidinone via $\mathrm{C}=\mathrm{N}$ double bond rotation at $\mathrm{T} \leq 250 \mathrm{~K}$, which is evidenced by the EXSY showing no exchange between the two diastereomers (Figure 4B). Since the intensity decrease is only observed for $\mathrm{H}_{1}$-exo-oxazolidinone, it implies the detected iminium ion is $Z$-configured (Figure $3 \mathrm{C}$ ).

Thus, CEST enables the first detection of an iminium ion intermediate in this enamine formation. In contrast, the selective $1 \mathrm{D}{ }^{1} \mathrm{H}$ EXSY for the same reaction mixture at the same conditions does not show any exchange peak at $9.12 \mathrm{ppm}$ on selective excitation of the exo-oxazolidinone peak (Figure $4 B$ ). Due to the low population of the iminium ion under these conditions, the sensitivity of EXSY or other conventional NMR methods is insufficient to detect the transient intermediates. The iminium ion observation only in CEST clearly highlights its potential in detection of transient reaction intermediates. Importantly it does not require any additional hyperpolarizing sources and hence gives information about intermediates without influencing the chemical systems. Additionally, the method is also possible in case of high spectral complexity (Figure 4C).

Population and Kinetics via CEST Profile. In the subsequent step, a complete CEST profile, that is, a plot of intensity change of exo-oxazolidinone peak vs chemical shift of selective saturation was generated to determine the population of the $Z$-iminium ion and to measure the rate of ring opening/ ring closing (Figure 5, circles). To access encoded information

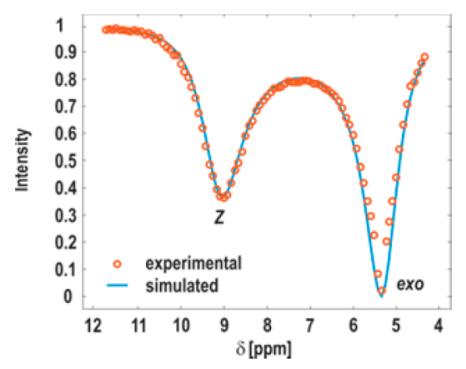

Figure 5. CEST profile at $250 \mathrm{~K}$; intensity variation of $\mathrm{H}_{1}$ exo peak on stepwise selective saturation over chemical shift. The major dip at 5.4 ppm is due to saturation of the $\mathrm{H}_{1}$ exo peak itself, and minor peak is due to saturation transfer from $Z$-iminium ion.

exchange rates and population ratio $[Z] /[$ exo $]$, a two site exchange CEST profile (Figure 5, blue curve) was simulated by using Bloch-McConnell equations, ${ }^{36-38}$ which correlates concentration of exchange species, longitudinal relaxation rates, and exchange rate constants (Supporting Information).

Subsequently, the simulated data were fitted to the experimental data (Figure 5) with a multiparameter optimization. The parameters concentration of exo-oxazolidinone $\left(M_{\text {exo }}\right.$ $=1)$ and the longitudinal relaxation times of oxazolidinone $\left(T_{\text {lexo }}\right)$ and $Z$-iminium ion $\left(T_{1 z}\right)$ are fixed, while varying the concentration of $Z$-iminium ion $\left(M_{\mathrm{imi}}\right)$ and the rate of ring closing $\left(k_{Z \rightarrow \text { exo }}\right)$. The longitudinal relaxation time $\left(T_{\text {lexo }}=1.8 \mathrm{~s}\right)$ of exo-oxazolidinone was obtained by an inversion recovery experiment while saturating the iminium ion resonance at 9.12 $\mathrm{ppm}$. The same $T_{1}$ value was assumed for the corresponding $Z$ iminium ion as well. The solutions of Bloch-McConnell equations and data fitting procedure are followed as mentioned in earlier reports ${ }^{37,54}$ and discussed in the Supporting Information. The obtained rates of exchange $k_{Z \rightarrow \text { exo }}, k_{\text {exo } \rightarrow Z}$ and the population of $Z$-iminium ion are reported in Table 1 . We could obtain a ring closing rate constant of $k_{Z \rightarrow \text { exo }} \approx 1300$ 
Table 1. Relative Populations and Rate Constants Measured from CEST and 1D EXSY

\begin{tabular}{cccccc} 
& \multicolumn{3}{c}{ CEST } & & 1D EXSY \\
\cline { 2 - 4 } temperature $(\mathrm{K})$ & {$[Z] /[$ exo $]$} & $k_{\text {z/exo }}\left(\mathrm{s}^{-1}\right)$ & $k_{\text {exo } / \mathrm{z}}\left(\mathrm{s}^{-1}\right)$ & & $k_{\text {exo } / \mathrm{z}}\left(\mathrm{s}^{-1}\right)$ \\
250 & 0.02 & $1300 \pm 50$ & $26 \pm 2$ & & n.a. \\
213 & 0.08 & $50 \pm 3$ & $4 \pm 1$ & & $2.15 \pm 1$ \\
\hline
\end{tabular}

$\mathrm{s}^{-1}$ and a ring opening rate constant $k_{\text {exo } \rightarrow Z} \approx 26 \mathrm{~s}^{-1}$ at $250 \mathrm{~K}$. The relative population collected from CEST is $Z /$ exo $\approx 0.02$, which corresponds to $+8.1 \mathrm{~kJ} / \mathrm{mol}$ free energy difference in favor for exo. The free energy barriers at $250 \mathrm{~K}$ calculated for ring closing and ring opening from the rates by using Eyring equation are +45.9 and $+54 \mathrm{~kJ} / \mathrm{mol}$, respectively. The energies obtained are in good agreement with our theoretical data $\left(\Delta G^{\ddagger}{ }_{268}=+42.2 \mathrm{~kJ} / \mathrm{mol} Z \rightarrow\right.$ exo and $+49.5 \mathrm{~kJ} / \mathrm{mol}$ for exo $\rightarrow$ $Z$ ). This quantitative information on iminium ions fills the missing blanks in enamine reaction pathway, assisting in better understanding.

Validation of CEST Method. The CEST measurements and its profile indicated that the population of the $Z$-iminium is still by far too low to be detected by conventional NMR ( 80 $\mu \mathrm{M})$. The traditional way to increase the population and minimize the transiency of the charge separated intermediate is to increase the dielectricity of the solvent by decreasing the measurement temperature. Indeed, at $213 \mathrm{~K}$, we could observe a 4-fold enhancement of the iminium intermediate population by CEST $(0.32 \mathrm{mM})$. The enhancement allows us also to detect the iminium species by conventional NMR (Figure 6A). Additionally, at this temperature we detected an exchange peak at $9.12 \mathrm{ppm}$ in the conventional $1 \mathrm{D}$ EXSY experiment by the selective excitation of the $\mathrm{H}_{1}$ exo-oxazolidinone peak (5.4 ppm; Figure 6B).

This gives us the opportunity to compare both CEST and 1D EXSY data for exchange rate measurements. Therefore, a series of 1D EXSY experiments were recorded with different mixing times (5-35 $\mathrm{ms}$ in $5 \mathrm{~ms}$ increment), and the obtained spectra are shown in Figure 6B. To determine the rate constant from the EXSY data, a plot of intensity of the $Z$-iminium peak vs mixing time is generated (Figure $6 \mathrm{~B}-\mathrm{C}$ ). It is known that in the initial linear buildup, the slope directly gives the rate of exchange from exo-oxazolidinone to $Z$-iminium ion. ${ }^{15}$ From the rate and the initial exo-oxazolidinone concentration $\left(I_{0}(\right.$ exo $\left.)\right)$, the rate constant for ring opening, $k_{\text {exo } / Z} \approx 2.15 \mathrm{~s}^{-1}$ is obtained (Figure 6C). The initial intensity $\left(I_{0}(\right.$ exo $\left.)\right)$ is obtained by back calculation from the spectra.

For comparison, CEST experiments were recorded at $213 \mathrm{~K}$ for the same mixture. The plotted CEST spectra are shown in Figure 6D. Again the rate constant and population of $Z$ iminium are extracted by comparing with simulated spectra from the Bloch-McConnell equations; the shown circles are experimental data and the curve represents a simulated profile. For simulating the CEST profile, a relaxation time $T_{1}=0.8 \mathrm{~s}$ was used for both exo-oxazolidinone and $Z$-iminium ion, which was obtained from an inversion recovery experiment for exooxazolidinone peak while on saturating at $9.12 \mathrm{ppm}$. The obtained rate constants and population are compared and are tabulated in Table 1 . At $213 \mathrm{~K}$, the obtained rate constants from 1D EXSY and CEST for ring opening are $k_{\text {exo } \rightarrow Z} \approx 2.15$ $\mathrm{s}^{-1}$ and $4 \mathrm{~s}^{-1}$ respectively. The values obtained from both methods are of the same magnitude, and hence it validates CEST(within experimental error 5-7\%). The reduced exchange rate at $213 \mathrm{~K}$ results in narrower line width for
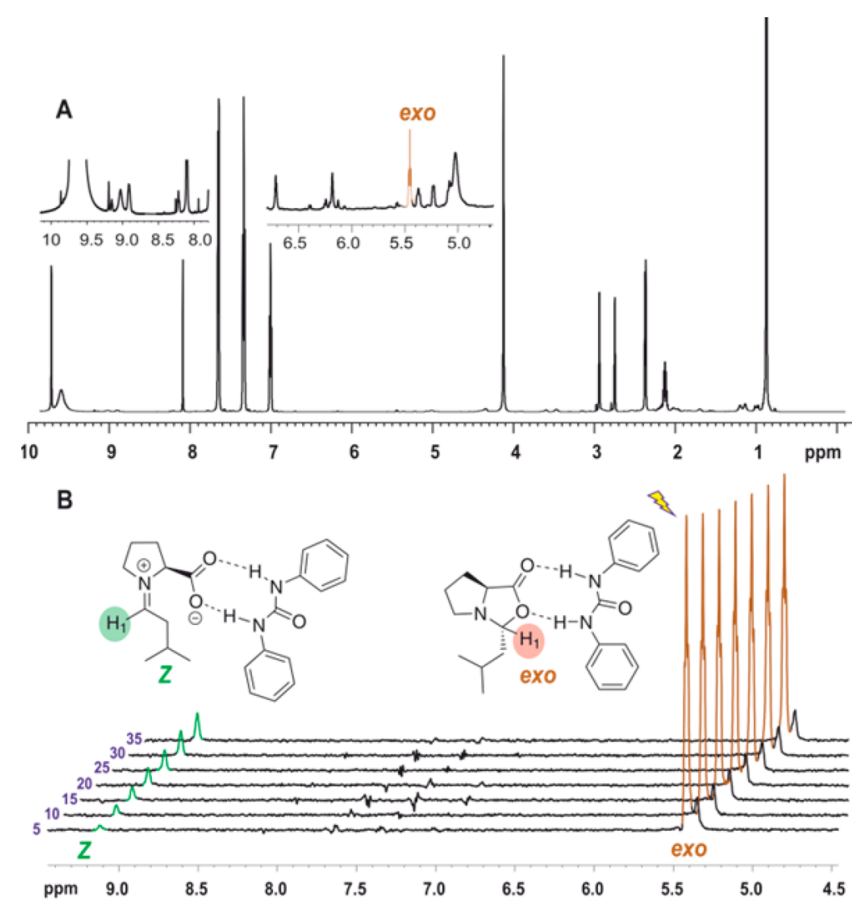

C

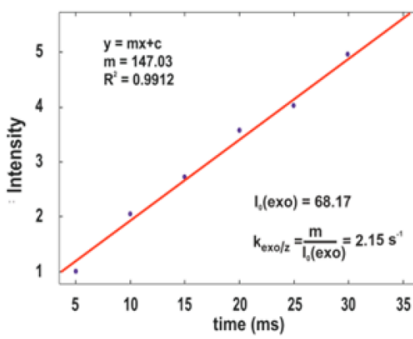

D

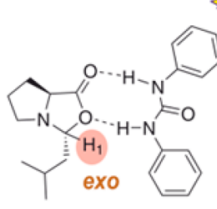

Figure 6. Normal $1 \mathrm{D}{ }^{1} \mathrm{H}$ spectrum showing two scaled regions around 5.5 and $9 \mathrm{ppm}$ at $213 \mathrm{~K}$ (A). 1D ${ }^{1} \mathrm{H}$ EXSY stacked spectra with different mixing time, in ms increments (B). Linear plot of intensity of iminium peak obtained from 1D EXSY vs mixing time for measurement of exchange rate (C). CEST experimental (circles) and simulated (curve) spectra at $213 \mathrm{~K}(\mathrm{D})$.

CEST profile (Figure 6D) compared to line widths in CEST profile at $250 \mathrm{~K}$ (higher exchange rates; Figure 5).

Even at $213 \mathrm{~K}, \mathrm{CEST}$ shows comparatively a stronger amplified signal for Z-iminium ion than in the 1D EXSY spectra. In addition CEST provides information about the concentration of $Z$-iminium ion, and both rate constants of ring closing $\left(k_{Z \rightarrow e x o}\right)$ and ring opening $\left(k_{\text {exo } \rightarrow Z}\right)$. In this case the obtained relative concentration of $Z$-iminium is $[Z] /[$ exo $] \approx$ 0.08 , rate constant for ring closing is $\approx 50 \mathrm{~s}^{-1}$, and rate constant for ring opening is $\approx 4 \mathrm{~s}^{-1}$. The corresponding activation barriers obtained for ring closing and ring opening are 44.1 and $49.1 \mathrm{~kJ} / \mathrm{mol}$, respectively. On the other hand $1 \mathrm{D}$ EXSY only gives the rate constant of ring opening $\left(k_{\text {exo } \rightarrow Z}\right)$. A difficulty in the integration of ${ }^{1} \mathrm{H}$ peak of $Z$-iminium ion in $1 \mathrm{D}{ }^{1} \mathrm{H}$ due to low intensity and overlap of other peaks (Figure 6A) restricts the extraction of population and rate constant $k_{Z \rightarrow \text { exo }}$. Furthermore, extraction of rate constants from 1D EXSY needs a series of experiments with varied mixing times and it needs to satisfy a linear relationship, which takes more time and is a serious problem particularly for low concentrated nuclei. This more often violates the linearity relationship between intensity buildup and mixing time. 
In summary, the experimental results gained by the application of CEST in combination with 1D EXSY and high-level theoretical calculations provide detailed free energy landscape of E,Z-iminium ions, endo as well as exooxazolidinone in enamine formation pathway (Figure 7). The
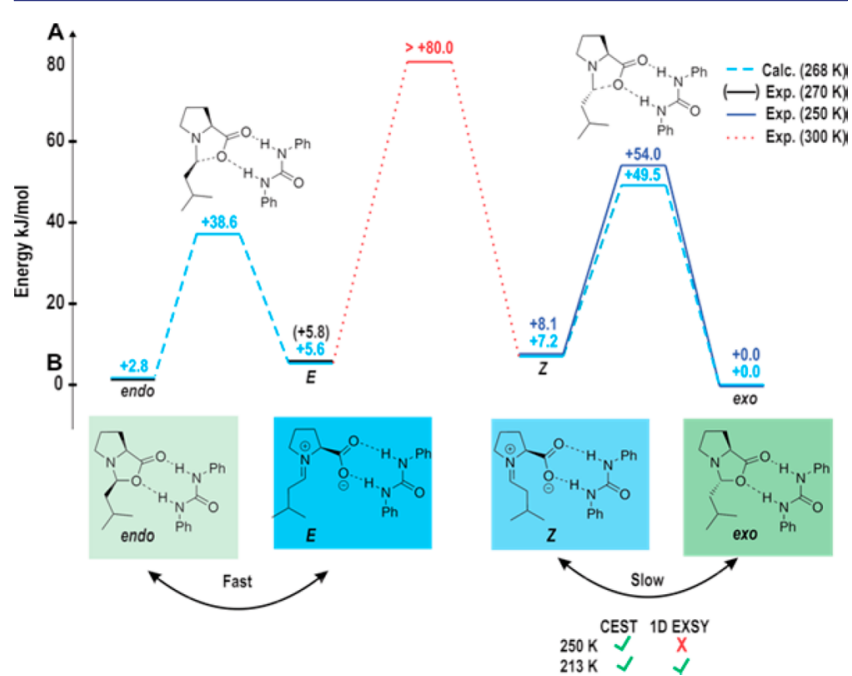

Figure 7. Compared thermal energy and free energy barriers obtained from experimental and theoretical calculations for endo-, exooxazolidinone, $E$ and $Z$-iminium. For the exo $\leftrightarrow$ endo exchange, the barrier was taken from the data at $300 \mathrm{~K}$. The relative energy of endo to exo could not be obtained at $250 \mathrm{~K}$, but was assumed as the similar to the theoretical value at $268 \mathrm{~K}$ (blue with dashed lines).

unprecedented detection of the $Z$-iminium ion intermediate, accessed free energies, and free energy barriers between $E \leftrightarrow$ endo (calculated) and $Z \leftrightarrow$ exo (experimental and calculated) verify our earlier theoretical calculations supporting the iminium pathway in the present system.

Computational Details. The geometry of all intermediates and transition states was optimized at TPSS-D3/def2-SVP level of theory in continuum (CPCM) of DMF. The dielectric constant of the solvent (DMF) was modified to mimic lowtemperature conditions of the NMR measurement $\left(\varepsilon_{268-215 \mathrm{~K}}=\right.$ 42.99-55.08). Nevertheless, the relative energy of the intermediates does not change significantly $(<1 \mathrm{~kJ} / \mathrm{mol})$ upon variation of dielectric constants. However, at low-temperature, theoretical calculations fail to predict correct populations of iminium ions due to difficulties in simulating the effect of dielectricity changes of polar solvents (DMF).

Subsequently thermochemical analysis was performed to identify unambiguously the minima (zero imaginary vibrational mode) and maxima (exactly one imaginary vibrational mode). Single point calculations at DLPNO-CCSD $(\mathrm{T}) / \mathrm{CBS}$ level of theory were added above the optimized geometry (for the extrapolation procedure see Supporting Information). ${ }^{55,56}$ The software used for optimization, frequency analysis, and NMR shifts calculation was Gaussian 09, D.01. ${ }^{57}$ For the single points ORCA 3.0.3 was employed. ${ }^{58}$ NBO6.0 was used for charge analysis. ${ }^{59}$

\section{CONCLUSION}

The study illustrates the high potential of the CEST method as a mechanistic tool to detect low populated intermediates (inaccessible to classical NMR methods), enabling new insights into the reaction pathway. In addition to the enhanced sensitivity, CEST provides thermodynamic and kinetic data of these intermediates in terms of population and exchange rates. The obtained detailed information about mechanistic steps between the intermediates assists in experimental validation of theoretically proposed mechanistic pathways. In the present mechanistic study of enamine formation, the CEST elucidates a possible mechanistic pathway by detecting and characterizing the previously missed in situ iminium ions. The obtained population and energy barriers pin pointed the iminium pathways and corroborated earlier theoretical predictions. Thus, CEST together with computational studies offer a better approach to probe missing intermediates in the reaction to pinpoint the possible mechanistic pathway.

In general, most of the chemical or catalytic reactions possess equilibria, which can be explored for application of CEST in mechanistic investigations. Further, the method can be combined with other sensitivity enhancement methods, like dissolution DNP, PHIP, or use of cryo probes, which can multiply the sensitivity to access ultralow (nano molar) concentrated intermediates.

\section{ASSOCIATED CONTENT}

\section{S Supporting Information}

The Supporting Information is available free of charge on the ACS Publications website at DOI: $10.1021 /$ jacs.7b12343.

Experimental details, NMR spectra, and computational details (PDF)

\section{AUTHOR INFORMATION}

\section{Corresponding Author}

*ruth.gschwind@ur.de

ORCID

Ruth M. Gschwind: 0000-0003-3052-0077

Notes

The authors declare no competing financial interest.

\section{ACKNOWLEDGMENTS}

We would like to thank the German Science Foundation (DFG grant GS 13/4-1) and the European Research Council (ERCCoG 614182 - IonPairsAtCatalysis) for financial support.

\section{REFERENCES}

(1) Greindl, J.; Hioe, J.; Sorgenfrei, N.; Morana, F.; Gschwind, R. M. J. Am. Chem. Soc. 2016, 138, 15965.

(2) Seegerer, A.; Hioe, J.; Hammer, M. M.; Morana, F.; Fuchs, P. J. W.; Gschwind, R. M. J. Am. Chem. Soc. 2016, 138, 9864.

(3) Sorgenfrei, N.; Hioe, J.; Greindl, J.; Rothermel, K.; Morana, F.; Lokesh, N.; Gschwind, R. M. J. Am. Chem. Soc. 2016, 138, 16345.

(4) Schmid, M. B.; Zeitler, K.; Gschwind, R. M. Angew. Chem., Int. Ed. 2010, 49, 4997.

(5) Frihed, T. G.; Bols, M.; Pedersen, C. M. Chem. Rev. 2015, 115, 4963.

(6) Zhang, L.; Ren, Y.; Yue, B.; He, H. Chem. Commun. 2012, 48, 2370.

(7) Lodewyk, M. W.; Siebert, M. R.; Tantillo, D. J. Chem. Rev. 2012, $112,1839$.

(8) Marigo, M.; Jørgensen, K. A. In Enantioselective Organocatalysis; Wiley-VCH Verlag GmbH \& Co. KGaA: Weinheim, Germany, 2007; pp 56-76.

(9) List, B. Synlett 2001, 2001, 1675.

(10) Mukherjee, S.; Yang, J. W.; Hoffmann, S.; List, B. Chem. Rev. 2007, 107, 5471. 
(11) Sharma, A. K.; Sunoj, R. B. Angew. Chem., Int. Ed. 2010, 49, 6373

(12) List, B.; Lerner, R. A.; Barbas, C. F., III J. Am. Chem. Soc. 2000, 122,2395 .

(13) Bahmanyar, S.; Houk, K. N.; Martin, H. J.; List, B. J. Am. Chem. Soc. 2003, 125, 2475.

(14) Seebach, D.; Beck, A. K.; Badine, D. M.; Limbach, M.; Eschenmoser, A.; Treasurywala, A. M.; Hobi, R.; et al. Helv. Chim. Acta 2007, 90, 425.

(15) Haindl, M. H.; Hioe, J.; Gschwind, R. M. J. Am. Chem. Soc. 2015, 137, 12835 .

(16) Schmid, M. B.; Zeitler, K.; Gschwind, R. M. J. Am. Chem. Soc. 2011, 133, 7065 .

(17) List, B.; Hoang, L.; Martin, H. J. Proc. Natl. Acad. Sci. U. S. A. 2004, 101, 5839.

(18) List, B. Acc. Chem. Res. 2004, 37, 548.

(19) Schmid, M. B.; Zeitler, K.; Gschwind, R. M. Chem. - Eur. J. 2012, $18,3362$.

(20) Schmid, M. B.; Zeitler, K.; Gschwind, R. M. Chem. Sci. 2011, 2, 1793.

(21) Ashley, M. A.; Hirschi, J. S.; Izzo, J. A.; Vetticatt, M. J. J. Am. Chem. Soc. 2016, 138, 1756.

(22) Gajan, D.; Bornet, A.; Vuichoud, B.; Milani, J.; Melzi, R.; van Kalkeren, H. A.; Veyre, L.; Thieuleux, C.; Conley, M. P.; Gruning, W. R.; Schwarzwalder, M.; Lesage, A.; Coperet, C.; Bodenhausen, G.; Emsley, L.; Jannin, S. Proc. Natl. Acad. Sci. U. S. A. 2014, 111, 14693.

(23) Bornet, A.; Maucourt, M.; Deborde, C.; Jacob, D.; Milani, J.; Vuichoud, B.; Ji, X.; Dumez, J. N.; Moing, A.; Bodenhausen, G.; Jannin, S.; Giraudeau, P. Anal. Chem. 2016, 88, 6179.

(24) Chen, C.; Shih, W.; Hilty, C. J. Am. Chem. Soc. 2015, 137, 6965.

(25) Duckett, S. B.; Mewis, R. E. Acc. Chem. Res. 2012, 45, 1247.

(26) Barskiy, D. A.; Shchepin, R. V.; Coffey, A. M.; Theis, T.; Warren, W. S.; Goodson, B. M.; Chekmenev, E. Y. J. Am. Chem. Soc. 2016, 138, 8080.

(27) Eshuis, N.; Hermkens, N.; Van Weerdenburg, B. J. A.; Feiters, M. C.; Rutjes, F. P. J. T.; Wijmenga, S. S.; Tessari, M. J. Am. Chem. Soc. 2014, 136, 2695.

(28) Lee, J. H.; Sekhar, A.; Cavagnero, S. J. Am. Chem. Soc. 2011, 133, 8062.

(29) Eisenreich, W.; Joshi, M.; Weber, S.; Bacher, A.; Fischer, M. J. Am. Chem. Soc. 2008, 130, 13544.

(30) Roth, H. D.; Manion Schilling, M. L. J. Am. Chem. Soc. 1980, 102, 4303 .

(31) Morris, J. I.; Morrison, R. C.; Smith, D. W.; Garst, J. F. J. Am. Chem. Soc. 1972, 94, 2406.

(32) Kiryutin, A. S.; Sauer, G.; Yurkovskaya, A. V.; Limbach, H.-H.; Ivanov, K. L.; Buntkowsky, G. J. Phys. Chem. C 2017, 121, 9879.

(33) Guivel-Scharen, V.; Sinnwell, T.; Wolff, S. D.; Balaban, R. S. J. Magn. Reson. 1998, 133, 36.

(34) Ward, K.; Aletras, A.; Balaban, R. J. Magn. Reson. 2000, 143, 79.

(35) Liu, G.; Song, X.; Chan, K. W. Y.; Mcmahon, M. T. NMR Biomed. 2013, 26, 810.

(36) McMahon, M. T.; Gilad, A. A.; Zhou, J.; Sun, P. Z.; Bulte, J. W. M.; van Zijl, P. C. M. Magn. Reson. Med. 2006, 55, 836.

(37) Woessner, D. E.; Zhang, S.; Merritt, M. E.; Sherry, A. D. Magn. Reson. Med. 2005, 53, 790.

(38) Zaiss, M.; Bachert, P. Phys. Med. Biol. 2013, 58, R221.

(39) Yuwen, T.; Sekhar, A.; Kay, L. E. Angew. Chem., Int. Ed. 2017, $56,6122$.

(40) Lee, J.-S.; Regatte, R. R.; Jerschow, A. J. Magn. Reson. 2012, 215, 56.

(41) Xu, X.; Lee, J. S.; Jerschow, A. Angew. Chem., Int. Ed. 2013, 52, 8281.

(42) Döpfert, J.; Witte, C.; Schröder, L. J. Magn. Reson. 2013, 237, 34.

(43) Zhao, B.; Hansen, A. L.; Zhang, Q. J. Am. Chem. Soc. 2014, 136, 20.

(44) Ling, W.; Eliav, U.; Navon, G.; Jerschow, A. J. Magn. Reson. 2009, 194, 29.
(45) Long, D.; Delaglio, F.; Sekhar, A.; Kay, L. E. Angew. Chem., Int. Ed. 2015, 54, 10507.

(46) Thorarinsdottir, A. E.; Du, K.; Collins, J. H. P.; Harris, T. D. J. Am. Chem. Soc. 2017, 139, 15836.

(47) Ling, W.; Regatte, R. R.; Navon, G.; Jerschow, A. Proc. Natl. Acad. Sci. U. S. A. 2008, 105, 2266.

(48) Cai, K.; Haris, M.; Singh, A.; Kogan, F.; Greenberg, J. H.; Hariharan, H.; Detre, J. A.; Reddy, R. Nat. Med. 2012, 18, 302.

(49) Vallurupalli, P.; Bouvignies, G.; Kay, L. E. J. Am. Chem. Soc. 2012, 134, 8148.

(50) Schmid, M. B. Ph.D. Thesis, University of Regensburg Regensburg-Germany, 2011.

(51) Lakhdar, S.; Tokuyasu, T.; Mayr, H. Angew. Chem., Int. Ed. 2008, 47, 8723.

(52) Grošelj, U.; Beck, A.; Schweizer, W. B.; Seebach, D. Helv. Chim. Acta 2014, 97, 751.

(53) El-Hamdouni, N.; Companyó, X.; Rios, R.; Moyano, A. Chem. Eur. J. 2010, 16, 1142.

(54) Zaiss, M.; Zu, Z.; Xu, J.; Schuenke, P.; Gochberg, D. F.; Gore, J. C.; Ladd, M. E.; Bachert, P. NMR Biomed. 2015, 28, 217.

(55) Riplinger, C.; Neese, F. J. Chem. Phys. 2013, 138, 34106.

(56) Riplinger, C.; Sandhoefer, B.; Hansen, A.; Neese, F. J. Chem. Phys. 2013, 139, 134101.

(57) Frisch, M. J.; Trucks, G. W.; Schlegel, H. B.; Scuseria, G. E.; Robb, M. A.; Cheeseman, J. R.; Scalmani, G.; Barone, V.; Mennucci, B.; Petersson, G. A.; Nakatsuji, H.; Caricato, M.; Li, X.; Hratchian, H. P.; Izmaylov, A. F.; Bloino, J.; Zheng, G.; Sonnenberg, J. L.; Hada, M.; Ehara, M.; Toyota, K.; Fukuda, R.; Hasegawa, J.; Ishida, M.; Nakajima, T.; Honda, Y.; Kitao, O.; Nakai, H.; Vreven, T.; Montgomery, J. A., Jr.; Peralta, J. E.; Ogliaro, F.; Bearpark, M.; Heyd, J. J.; Brothers, E.; Kudin, K. N.; Staroverov, V. N.; Kobayashi, R.; Normand, J.; Raghavachari, K.; Rendell, A.; Burant, J. C.; Iyengar, S. S.; Tomasi, J.; Cossi, M.; Rega, N.; Millam, J. M.; Klene, M.; Knox, J. E.; Cross, J. B.; Bakken, V.; Adamo, C.; Jaramillo, J.; Gomperts, R.; Stratmann, R. E.; Yazyev, O.; Austin, A. J.; Cammi, R.; Pomelli, C.; Ochterski, J. W.; Martin, R. L.; Morokuma, K.; Zakrzewski, V. G.; Voth, G. A.; Salvador, P.; Dannenberg, J. J.; Dapprich, S.; Daniels, A. D.; Farkas, Ö.; Foresman, J. B.; Ortiz, J. V.; Cioslowski, J.; Fox, D. J. Gaussian 09; Gaussian, Inc.: Wallingford, CT, 2009.

(58) Neese, F. Wiley Interdiscip. Rev. Comput. Mol. Sci. 2012, $2,73$.

(59) Glendening, E. D.; Badenhoop, J. K.; Reed, A. E.; Carpenter, J. E.; Bohmann, J. A.; Morales, C. M.; Landis, C. R.; Weinhold, F. NBO 6.0; Theoretical Chemistry Institute, University of Wisconsin: Madison, WI, 2013. 